\title{
Diversity Sizing - How Different or Similar are European Women and Men?
}

\author{
Magdalena MANDALKA, Anke RISSIEK \\ Avalution $\mathrm{GmbH}$, Kaiserslautern, Germany \\ https://doi.org/10.15221/21.54
}

\begin{abstract}
The diversity discussion gets more and more important in our society and also the industry and body related product development are affected by this trend. For the apparel sector the diversity approach comes along with certain questions considering the feasibility of combining different genders in sizing. The following analysis focusses on differences and similarities of European female and male body dimensions and evaluates the question if both traditionally used single target customer groups can be combined to a gender-neutral target market and therefore consider the increasing importance of gender diversity. Average body measurements give an overview of how different average gender specific measurements are. Further analysis of measurement correlations visualize how female and male measurement distributions are differing or overlapping each other. The analysis of proportions and the generation of avatars gives an overview of what needs to be regarded within a possible diverse sizing approach and shows how gender diversity can be combined and implemented into apparel specific product development.

Average girth measurements already differ with regards to one gender within the European market. Adding an additional gender layer to the analysis leads to even more difference. Whereas average girth measurements and girth related body proportions can be covered by a feasible size range and fit tolerances, the differences in length dimensions represent the most challenging differences between both genders. The more overlapping the body proportions are, the easier the combination of both target groups is. The results show that a combination of both genders is possible, but it also leads to some length and fit related compromises in product quality.
\end{abstract}

Keywords: Diversity, sizing, gender, unisex, body measurements, correlations, iSize, proportions, fit, shape, apparel, size table

\section{Introduction}

Today the idea of genders is different to how it was generations before. The understanding of genders gets redefined in our society and the differences between "female" and "male" blurry more and more. Many industrial sectors already develop gender-neutral products that are completely independent from the gender of a person. As long as the product is related to single parts of the human body and the differences between average female and male measurements are not too big or do not lead to a reduction of product quality (e.g. for helmets, interior), this approach is very feasible. The expression "unisex" usually refers to such gender-neutral products. With regards to the apparel sector, this normally goes hand in hand with products that have huge fit tolerances and that are practical but not fashionable. Especially in the workwear sector, unisex products are quite common. Now, also the fashion industry faces this current gender revolution. As a result, more and more fashionable and diverse products are offered within collections to follow this huge trend in society [1,2].

In former times, apparel companies had to face the challenge of combining the proportions and average measurements within different age groups or markets $[3,4]$, but always with regards to only one single gender.

The development of diverse products or collections leads to a progressive and innovative approach. At the same time, the idea of combining both genders and with that regard the different morphological attributes, average measurements and different proportions of women and men is not only new but also challenging. There are open questions with regards to the sizing structure. The number of sizes, the appropriate labelling, the differences with regards to body measurements and the optimal height concept are just some of the questions that come along the approach of developing a diversity sizing concept.

The following analysis therefore focusses on the available differences and similarities between European women and men with regards to their body measurements and proportions. Based on the results, the question will be answered, if a diversity sizing approach is feasible and what needs to be regarded within the combination of both genders. The collected findings finally give a preview of what companies need to be aware of if they develop and offer gender-neutral products in the European market. 


\section{Methodology, infrastructure and test sample}

The gender specific analysis is done in Avalution's iSize portal, which was set up in 2009 after the SizeGERMANY size survey [5,6]. Today, many different global data pools with comparable body measurements according to ISO 8559 [7] and ISO 7250 [8] are implemented and available for analysis. The iSize portal allows the flexible combination of different target groups such as different age groups, markets or genders. Based on the target group definition, measurement analysis and also analysis that is related to the apparel industry, for example size table analysis and optimization or market share calculation can be done within the portal. For the following analysis, women and men between 18 and 65 years from the different European markets France, Italy, Germany and the Netherlands are regarded and compared. As especially the differences between the genders should be compared to each other, additionally all female European data and all male European data were combined to two separate gender specific data pools, "Europe women" and "Europe men". The creation of this data pools enables the optimal comparison between the genders.

\section{Analysis and results}

As the procedure and the content of the analysis refers to the structure and methodology of sizing systems in apparel industry, specific analysis approaches and measurements are selected. The analysis of European women and men exemplarily focusses on the main measurements, chest, waist and hip girth and the length dimensions, body height, arm length and inside leg length and points out differences and similarities of one-dimensional measurements. Additionally, those measurements are put into a relation with each other for further evaluation of measurement correlations and distributions. The analysis of proportions will finally clarify if a diverse sizing is reasonable within the regarded EU market.

\subsection{Average measurements}

Average body measurements already differ within one single gender in the European market. For companies that develop products that are related to the human body it is the challenge to find the best possible fit compromise without having too big product ranges or a too complex sizing system.

The single markets France, Germany, Italy and the Netherlands are analyzed with regards to average body dimensions, separate for women and men. Afterwards, the smallest and the biggest average values of each measurement are filtered out to get an overview about the variance of average measurements between one gender in Europe. Table 1 and 2 exemplarily show the minimum and maximum average values of the main body measurements chest, waist and hip girth and the length specific measurements body height, arm length and inside leg length. Additionally, the resulting differences between the minimum and the maximum values are documented to show the measurement variance within the one-gender market.

Table 1. Differences of average body measurements of European women between 18 and 65 years

\begin{tabular}{lccc}
\hline \multicolumn{1}{c}{ [cm] } & MAX average & MIN average & Difference \\
Chest girth & $100,8(\mathrm{NL})$ & $93,4(\mathrm{FR})$ & 7,4 \\
Waist girth & $85,7(\mathrm{NL})$ & $79,2(\mathrm{FR})$ & 6,5 \\
Hip girth & $106,3(\mathrm{NL})$ & $99,9(\mathrm{FR})$ & 6,4 \\
Body height & $167,5(\mathrm{NL})$ & $159,7(\mathrm{IT})$ & 7,8 \\
Arm length & $58,4(\mathrm{NL})$ & $55,8(\mathrm{IT})$ & 2,6 \\
Inside leg length & $78,8(\mathrm{NL})$ & $71,4(\mathrm{IT})$ & 7,4 \\
\hline
\end{tabular}


Table 2. Differences of average body measurements of European men between 18 and 65 years

\begin{tabular}{lccc}
\hline \multicolumn{1}{c}{ [cm $]$} & \multicolumn{3}{c}{ MEN } \\
Chest girth & $106,1(\mathrm{GE})$ & MIN average & Difference \\
Waist girth & $94,2(\mathrm{GE})$ & $102,0(\mathrm{FR})$ & 4,1 \\
Hip girth & $103,0(\mathrm{GE})$ & $89,0(\mathrm{FR})$ & 5,2 \\
Body height & $181,0(\mathrm{NL})$ & $99,9(\mathrm{FR})$ & 3,1 \\
Arm length & $64,0(\mathrm{NL})$ & $172,4(\mathrm{IT})$ & 8,6 \\
Inside leg length & $84,1(\mathrm{NL})$ & $61,5(\mathrm{IT})$ & 2,5 \\
\hline
\end{tabular}

Dutch women have the biggest average girth and length dimensions. In contrast to this, the French women have the smallest average girth measurements, whereas the Italian women are the shortest population. Also within the male market, the French men have the smallest average girth measurements, whereas the German men have the biggest average girth dimensions. With regards to the length specific measurements, the Italian men are the shortest and the Dutch men are the longest male population in the analysis.

In general, the analysis shows that European women have a bigger variance between their average measurements compared to European men. With regards to the girth measurements, for women the variance between the country specific average measurements is between $6,4 \mathrm{~cm}$ and $7,4 \mathrm{~cm}$. For men, the variance between the country specific average girth measurements within the different European markets is between $3,1 \mathrm{~cm}$ and $5,2 \mathrm{~cm}$. Especially, average length dimensions are very different within one gender. As an example, the spread in average body height between Dutch (maximum average) and French women (minimum average) is $7,8 \mathrm{~cm}$. For men, the difference between the shortest and the tallest average is even $8,6 \mathrm{~cm}$. As the other length dimensions arm lengths and inside leg length are shorter measurements absolutely seen, also the resulting differences are smaller.

Today the industry already uses certain strategies to cover the existing differences within one gender in the European market. If the range of measurements that needs to be covered is too wide for one single product, different sizes of the product with regards to width or length are offered. Especially the combination of different length and width options at the same time leads to improved market coverage [9]. Also products that show a certain fit tolerance or enable an adjustment of width and length based on the product design are feasible tools to cover an international market. Especially, the apparel industry also offers products that are related to different figure types to cover different body proportions and measurements along the markets [10].

If both genders are compared to each other, the difference between average measurements increases. Table 3 visualizes the resulting average measurements and differences between all female and male Europeans.

Table 3. Resulting measurement differences of European women and men between 18 and 65 years

\begin{tabular}{lccc}
\hline \multicolumn{3}{c}{ DIVERSITY COMPARISON } \\
\hline \multicolumn{1}{c}{$[\mathrm{cm}]$} & MALE Average & FEMALE Average & Difference (MAX - MIN) \\
Chest girth & 104,6 & 96,6 & 8,0 \\
Waist girth & 92,4 & 82,9 & 9,5 \\
Hip girth & 101,6 & 101,7 & 0,1 \\
Body height & 176,8 & 163,8 & 13,0 \\
Arm length & 62,7 & 57,1 & 5,6 \\
Inside leg length & 80,2 & 74,2 & 6,0 \\
\hline
\end{tabular}


In general, men have a bigger average chest girth compared to European women. The resulting difference between the average European female and male chest girth is $8,0 \mathrm{~cm}$. The difference is comparable to the differences that can be found within the European females $(7,4 \mathrm{~cm}$, table 1$)$, but in comparison to the European males $(4,1 \mathrm{~cm}$, table 2$)$ the variance with regards to the chest girths has increased by $95 \%$. Men also have a bigger average waist girth compared to women. The difference between the waist girths of women and men increases compared to the former one-gender analysis. If the European average female and male waist girths are compared, the resulting difference is $9,5 \mathrm{~cm}$. The difference between the genders is more than $46 \%$ bigger than the difference within the European females and nearly $83 \%$ bigger compared to the difference within the European males. At the same time, the average hip girths are nearly equally for women and men.

Also with regards to the length dimensions, body height and arm length, the differences are increasing, if the average measurements of both genders are compared to each other. In general men have much longer length dimensions compared to European females. For the arm length the difference between the female and male average is more than twice as big than within the one-gender comparisons.

The comparison of the average female and male body height now shows a difference of $13,0 \mathrm{~cm}$, which is more than a $51 \%$ bigger difference compared to the variance of the European male body heights (table 2, 8,6 cm). With regards to the average female body heights, which show a variance of $7,8 \mathrm{~cm}$ within the one-gender analysis, this even means an increase by nearly $67 \%$. At the same time, the difference between the average inside leg lengths of women and men is slightly smaller compared to the one-gender analysis and the different European markets.

In general, the analysis shows that with the comparison of women and men the resulting differences between average measurements tend to get bigger in contrast to one-gender measurement analysis, but not necessarily all differences increase. Some measurements and resulting differences are strongly related to gender specific differences, whereas other measurements are more connected to the measurement differences in the various European markets. The different strategies that are used to serve and cover international one-gender markets today, could also represent feasible starting points for the development of a diverse sizing.

\subsection{Correlations and measurement distributions}

Correlations visualize the relation and the distribution between two regarded measurements. They show, how one measurement behaves, if another measurement increases or decreases. Dependencies and independencies of measurements can be easily shown based on correlation diagrams. With regards to sizing, measurement correlations are especially important as the correlations also influence the final measurement concept of a product. The optimal relation between measurements is indispensable for optimal product fit, comfort and market coverage.

Therefore, the following analysis focusses on female and male measurement correlations and distributions and validates if both genders show similarities as this indicates, whether a common diverse sizing is feasible or not. With regards to girth measurements, the correlations and distributions between the chest girth and the measurements waist and hip girth are analyzed. With respect to the length dimensions, the correlations and distributions between the body height and the arm length is regarded. The results of the calculated correlations are documented and interpreted in table 4 and 5 , separately for women and men.

Table 4. Exemplarily analysis of female correlations within the European market, between 18 and 65 years

\begin{tabular}{|l|l|l|}
\hline WOMEN & Chest Girth & Interpreted correlation \\
\hline Waist Girth & 0,9 & High \\
\hline Hip Girth & 0,8 & High \\
\hline & Body Height & Interpreted correlation \\
\hline Inside Leg Length & 0,9 & Very high \\
\hline Arm Length & 0,8 & High \\
\hline
\end{tabular}


Table 5. Exemplarily analysis of male correlations within the European market, between 18 and 65 years

\begin{tabular}{|l|l|l|}
\hline MEN & Chest Girth & Interpreted correlation \\
\hline Waist Girth & 0,9 & High \\
\hline Hip Girth & 0,8 & High \\
\hline & Body Height & Interpreted correlation \\
\hline Inside Leg Length & 0,9 & High \\
\hline Arm Length & 0,8 & High \\
\hline
\end{tabular}

One of the main chances for the development of a diverse sizing are those areas within the correlation diagrams that show certain overlaps of female and male data. In between the area where the female and male point clouds are overlapping, persons with female and male identities have similar body measurements and proportions. The bigger the overlapping areas are, the bigger the potential of the diverse market and the chance for a diversity sizing approach is. Fig. 1 shows the correlation diagram of the girth measurements chest and hip girth, Fig. 2 describes the correlation between chest and waist girth.

The chest girth is always placed on the $x$-axis and the second measurement is placed on $y$-axis. The coral-colored point cloud represents females in Europe. The turquoise point cloud visualizes the male population from Europe. The grey color in the diagram marks the overlapping area between both genders. The darker lines in the correlation diagrams highlight the P5 and P95 of each measurement. Within the P5 and P95 area of both regarded measurements, $80 \%$ of the market is located. For the analysis with respect to the diversity sizing approach especially this $80 \%$-market represents a relevant benchmark. Diverse products need to fit optimally for females and males within this specific area. If one single product is not able to cover this area properly, compromises with regards to the product concept or solutions as additional product sizes need to be evaluated.

For both genders the correlations between the regarded measurements can be interpreted as high. If the chest increases, also the waist- and hip girth increase due to the strong dependencies between the measurements.

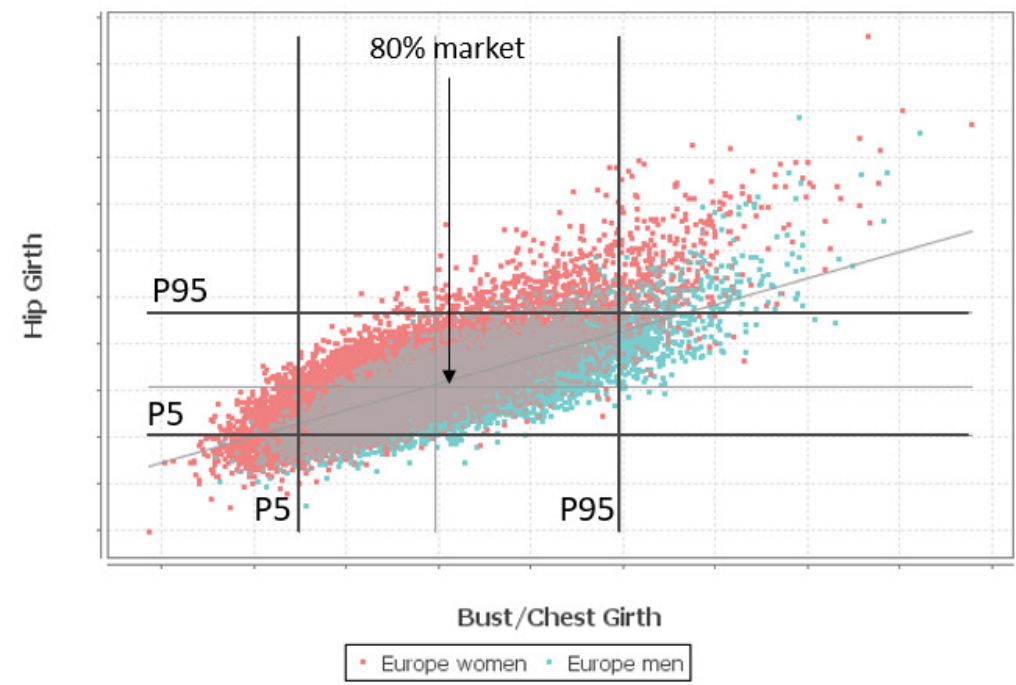

Fig. 1. Correlation between chest girth and hip girth of European women and men, between 18 and 65 years 


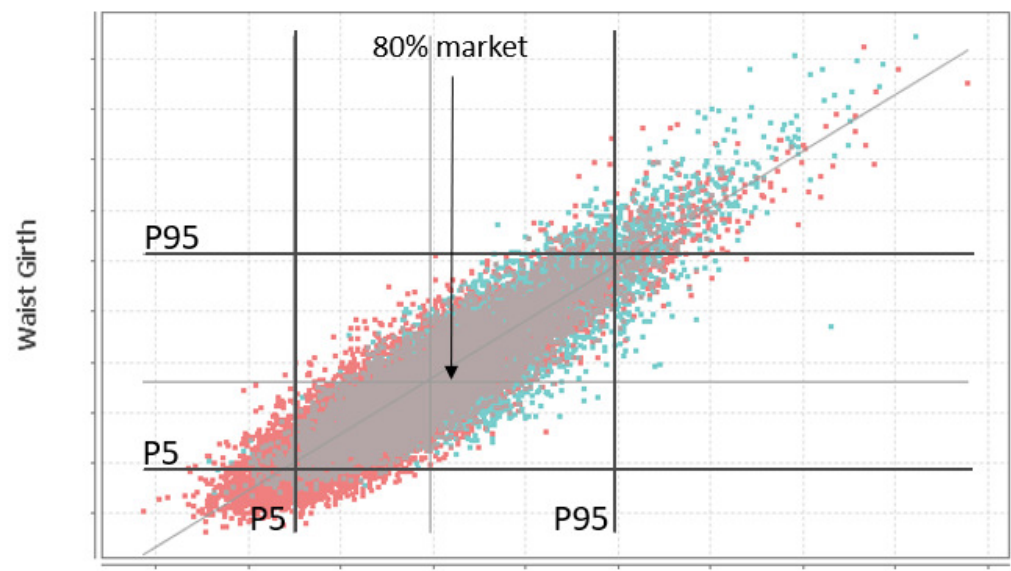

Bust/Chest Girth

Europe women * Europe men

Fig. 2. Correlation between chest girth and waist girth of European women and men, between 18 and 65 years

With regards to the correlation of chest- and hip girth, both point clouds are slightly shifted away from each other. Women tend to have bigger hips, whereas men with a comparable chest girth have smaller hip girths. Besides these differences, there is also an area within the relevant $80 \%$-market where both point clouds are overlapping each other with regards to their hip girths.

For the diverse sizing approach this indicates certain opportunities and challenges. A compromise in sizing for both genders with regards to the hip girth can cover a certain part of the distribution of female and male hip girths at the same time. For women with smaller hip girths and for men with broader hips a common size systematic can be worked out. At the same time, the compromise will not be able to cover both genders optimally. The more extremer female and male body types, for e.g., women with bigger hip girths and curvier proportions and men with relatively narrow hips compared to the average men, will probably not be covered by a diverse sizing approach.

The correlation diagram of chest and waist girth shows that women and men have a very similar measurement distribution. The female and male point clouds are nearly completely overlapping each other. For the very small chest girths, there is only female data available, but this area is mainly located out of the $80 \%$-area and therefore out of scope. For the diverse sizing approach this indicates an opportunity with regards to upper body products. As the distribution of female and male waist girths is similar in smaller and in bigger chest girths, the needed compromise in sizing will be rather small along the complete size range and still both genders can be covered properly.

In contrast to the girth measurements, the bigger challenge is to cover the different length specific measurement distributions and correlations of women and men. Fig. 3 shows the correlation between chest and body height. The chest girth is placed on the $x$-axis and the body height is positioned on the $y$-axis.

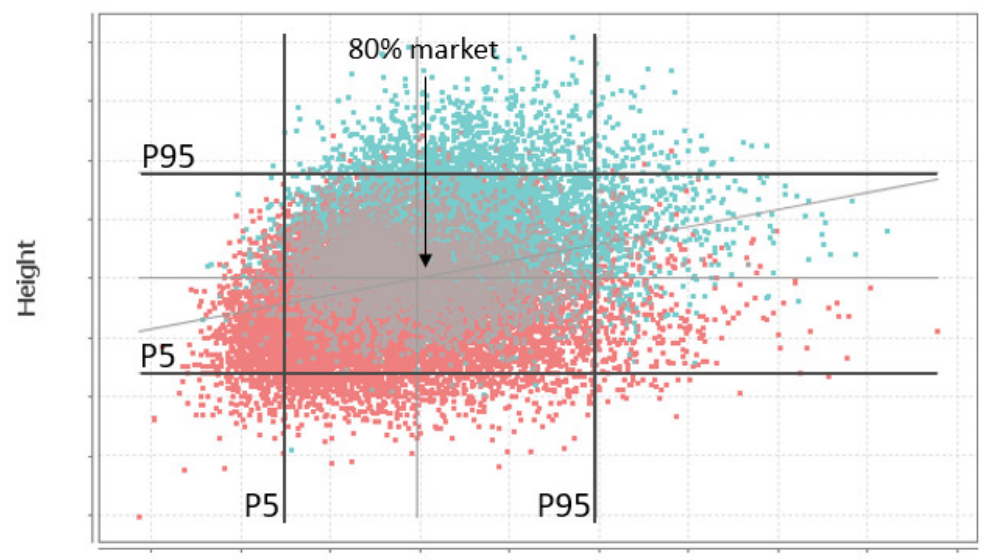

Bust/Chest Girth

- Europe women - Europe men

Fig. 3. Correlation between chest girth and body height of European women and men, between 18 and 65 years 
Due to the very different average body heights, the point clouds are shifted away from each other. The overlapping area between female and male data is comparably small. There are areas where only female or only male data is located within the correlation diagram. The range of available body heights between P5 and P 95 is about $30,0 \mathrm{~cm}$. For the diverse product development, this wide range will be very challenging to cover. An alternative approach could consider only the overlapping area of female and male data as a compromise for the diverse sizing and to keep the complexity of the needed sizing structure rather low.

Although the average length dimensions are very different between European women and men the general behavior of the length specific dimensions is very similar. The correlation between body height and the arm length can be interpreted as high for both genders. If the body height increases, male and female arm lengths increase in a comparable way. Fig. 4 shows that both point clouds are in line with each other.

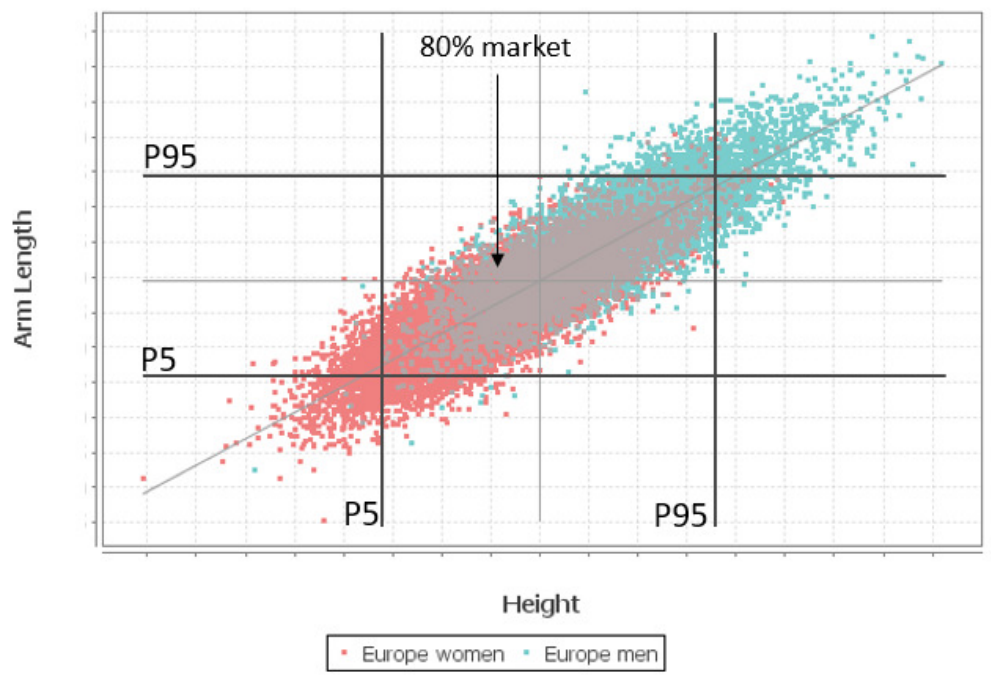

Fig. 4. Correlation between body height and arm length of European women and men, between 18 and 65 years

The grey area again highlights the overlapping data of women and men. There are areas within the $80 \%$-market, where only female or only male data is located. With regards to diverse product development, it needs to be evaluated, if focusing only on the overlapping area already leads to satisfying results or if the diverse products also reach the single-gender areas of the $80 \%$-market to increase the resulting market coverage. Also for other length dimensions this effect can be seen. As the analysis has already proven how different especially average female and male length dimensions are, an optimal length concept will be indispensable. The comparable measurement correlations and the overlapping areas of female and male data are advantages for the diversity sizing approach as it indicates the feasibility for combining both data pools.

\subsection{Measurement proportions}

Besides average measurements and correlations, body proportions are an important factor within the diversity sizing approach. Only if the combination of all regarded measurements is able to describe female and male bodies at the same time, a diverse sizing with a feasible sizing structure is possible. Fig. 5 visualizes how waist and hip girth are developing during a constant increase of the chest girth. The analysis is done separately for women and men. For the increase of the chest, exemplarily $5,0 \mathrm{~cm}$ steps are selected. The chosen chest girth range matches the P5 and P95 of the chest girth within the regarded target group. For each chest girth value, the average female and male hip- and waist girths are calculated. Afterwards the resulting values are compared and differences between those values within one chest girth category are calculated for further analysis. 


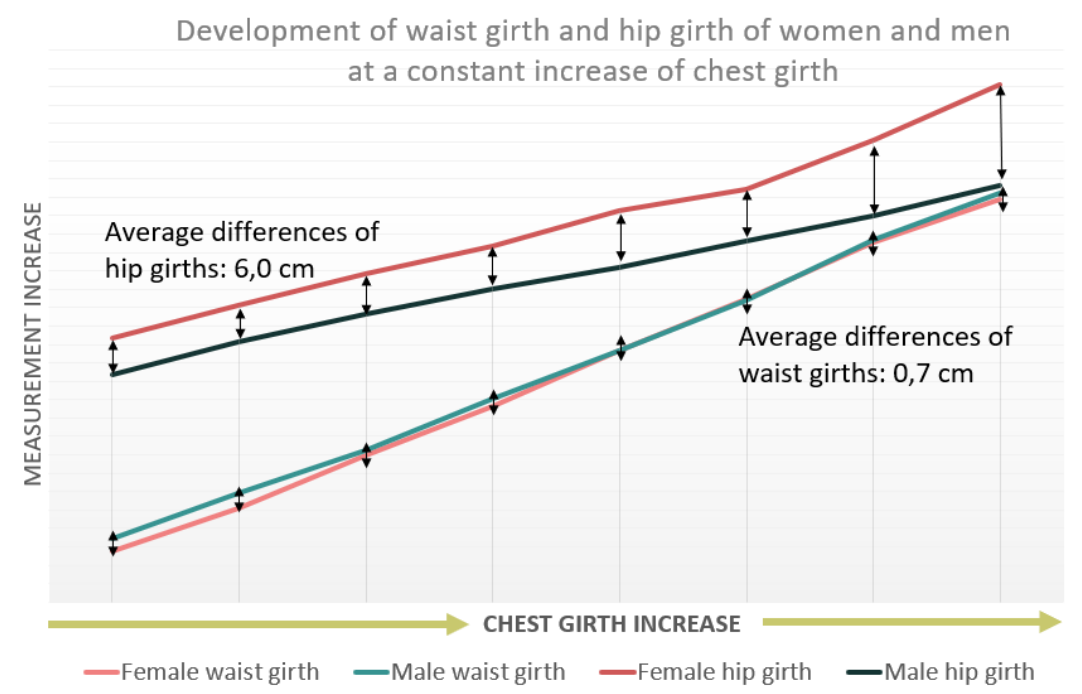

Fig. 5. Development of waist girth and hip girth at a constant increase of chest girth, European women and men between 18 and 65 years

Comparable to the correlation analysis the diagram shows that with the same chest girth men and women have nearly identical waist girths. The average difference between the calculated waist girths within each chest category is about $0,7 \mathrm{~cm}$.

With an average difference of $6,0 \mathrm{~cm}$, the average hip girths of women and men are much more different. Within the smaller chest girth categories, the average hip girths are closer together than in the bigger chest categories. Within the smaller chest girths, the difference between the calculated hip girths is about $4,0 \mathrm{~cm}$. With increasing chest, the hip girths of women and men get more and more different. The reddish line shows that women have a much stronger increase of the hip girth than men (dark green line). In bigger sizes the difference between female and male hip girths is about $11,0 \mathrm{~cm}$.

This exemplary overview already indicates certain preconditions for the diversity sizing. With regards to the upper body, a diverse sizing seems feasible. The development of a diverse sizing structure with products in different sizes but suitable for both genders could be developed based on the regarded measurements chest and waist girth. The main challenges can be seen in the lower body and especially in the area of the bigger girth dimensions due to the very different hip girth developments.

The results might indicate that a diverse sizing with regards to women and men is limited to a certain range of girth dimensions. If also people with bigger girth measurements should be served within the diverse sizing concept, increased product tolerances are required to cover female and male body proportions. Increased fit tolerances with regards to the hip area will be indispensable to generate the best possible compromise. Products and fits that are very tight fitted to the body might be out of scope or at least very challenging for the diversity sizing approach. Also a separation between upper and lower body as compromise could be a feasible approach.

Fig. 6 focusses on the differences of length proportions between women and men in Europe. The main length measurement, the body height, is positioned on the horizontal axis and a $5,0 \mathrm{~cm}$ scaling is selected to visualize the range of different body heights according to the P5 and P95 of the diverse market. The average arm length and inside leg length of women and men within each body height category is calculated and applied within the diagram. Additionally, the resulting average differences between the values of women and men in each body height category are calculated.

The development of the length dimensions within an increasing body height is similar for both genders. Also along the complete range of different body heights, the differences between women and men are very constant. Absolutely seen the inside leg length is a longer dimension compared to arm length. Therefore, the differences between women and men are slightly bigger for the inside leg length than for the arm length. 


\section{Development of arm length and inside leg length of women and men at a constant height increase}

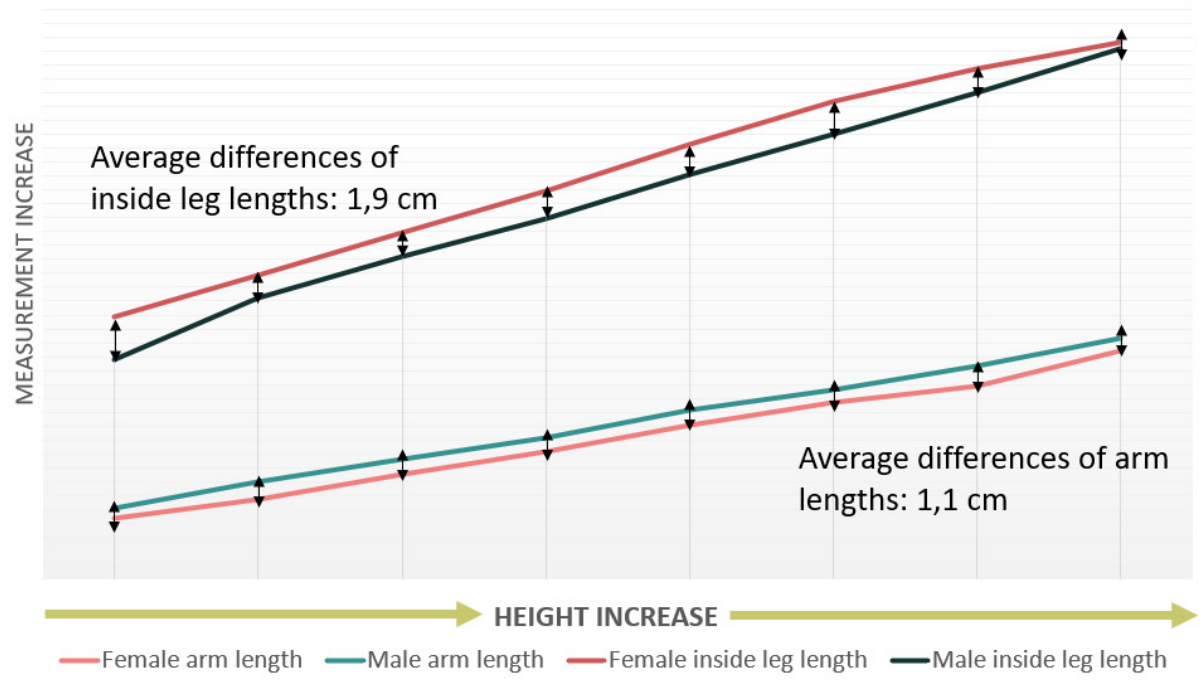

Fig. 6. Development of arm length and inside leg length at a constant height increase, European women and men between 18 and 65 years

The inside leg length differs around $1,9 \mathrm{~cm}$ for men and women. For the arm length the differences between the female and male values with regards to one body height is only around $1,1 \mathrm{~cm}$. This shows that although the average values of women and men are totally different, the behavior of the length dimensions within one single body height is very comparable. As men and women have comparable length proportions in general, diverse products can easily cover both genders at the same time as long as one height category is regarded.

Avatars are common tools for virtual body related product development. They are used in various branches and also with regards to the diversity sizing approach, it offers certain opportunities for fit evaluation and testing [11]. Fig. 7 illustrates female and male avatars that are generated based on the same measurement set. On the left side an avatar is generated based on female scan data. In the middle, an avatar based on male scan data is illustrated. As measurement set, the average measurements of the combined diverse market are calculated and applied.

Both avatars point out the resulting fit compromise. The female avatar is comparably straight in the area of the hip, whereas the male avatar has slightly curvier hips compared to other male standard avatars. At the same time both avatars are fully proportional. Although the diverse sizing leads to a general fit compromise, none of the avatars shows artificial or critical parts of the body due to the diverse measurement set. Additionally, the overlay of both avatars on the right side of Fig. 7 proves the matching of both shapes. The biggest difference can be seen in the area of the chest. Due to the development of the female chest, there are some differences between both avatars. In general, the side view of the overlay shows the main differences in the front of both torsos. All other parts of the body such as the back area for example are comparable for women and men. 


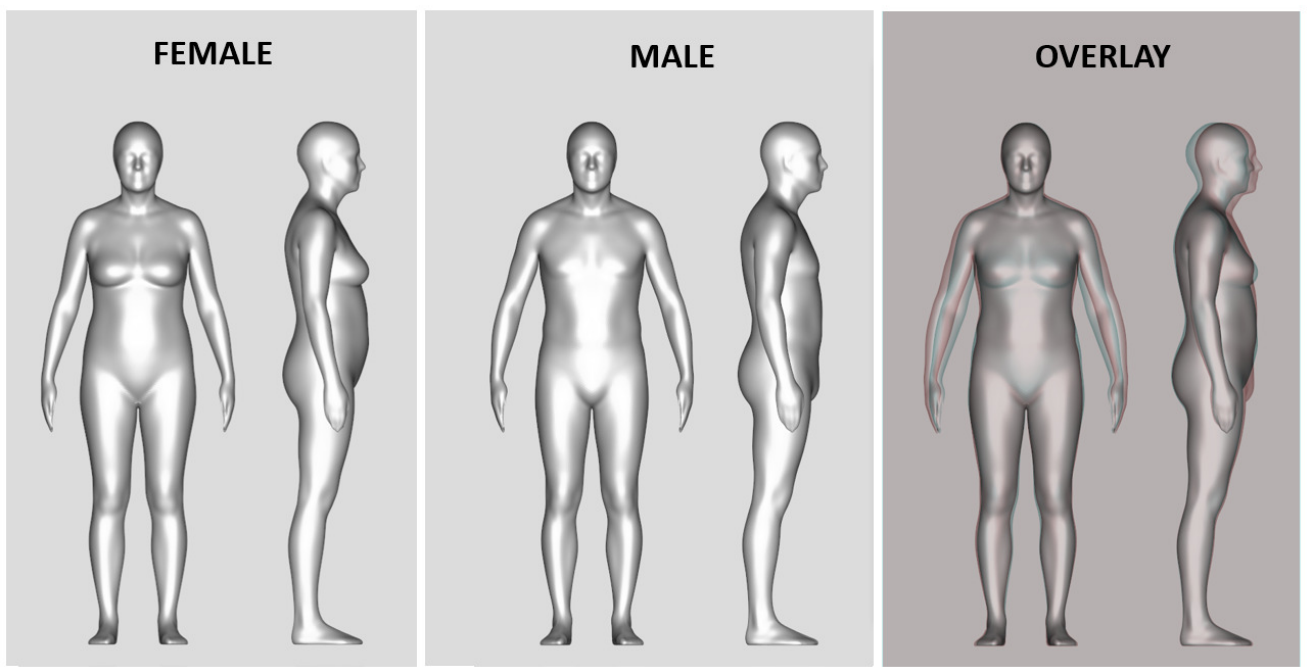

Fig. 7. Diverse avatars generation. Female (left) and male (middle) avatar generated based on same measurements and additional overlay of both avatars (right)

With regards to the divers sizing approach the illustration of both avatars shows that both genders can be combined within product development. The visible differences of women and men with the same measurement set will be covered by product ease and fit tolerances. Avatars have a key position in the diverse sizing approach. Female and male avatars with the same measurement set can be a significant support during the diversity sizing approach and support product development processes.

\section{Summary and outlook}

Diversity is a huge trend in our society and especially also in apparel product development. At the end of the analysis the basic findings are summarized, and possible next steps based on the results are described to follow this enormous trend and to show how diversity sizing could finally be applied in the fashion industry. Other possible analysis approaches in the field of diversity analysis are also mentioned to show the huge range of different options and potentials.

\subsection{Summary}

The analysis has shown that in general women and men have different average measurements, but already within one single gender, measurements differ in between the European markets. The industry, such as the apparel sector, already has various strategies to manage those differences for serving international one-gender markets.

With regards to girth measurements the analysis of correlations, distributions and proportions has shown that both genders can be combined within a diverse market. The measurement correlation of men and women are very similar, whereas measurement distributions are not identical but also comparable. Those areas, where male and female data overlap each other, can be used for a diverse product development. The analysis also points out that not all people with female and male identities will be covered by a diverse sizing approach as sizing systems are always linked to compromises. Those extremer female proportions that are very curvy or those male proportions that show very narrow hips in relation to the chest, will not be covered by a diversity sizing approach. Also within the bigger girth dimensions the combination of female and male data is more challenging as women and men tend to get more and more different in the area of the lower body.

Although women and men have comparable length proportions and measurement relations, the main challenge for a diversity sizing is the huge difference between average female and male length dimensions. With regards to length specific measurements, only compromises can be achieved.

\subsection{Implementation of a diversity sizing}

Based on the shown analysis, the development of a final diversity approach that covers both genders in a proper way and leads to fashionable products, is the next logical step. To finally achieve a sizing that covers women and men at the same time, different aspects need to be regarded and finally developed. Figure 8 gives an overview about different aspects for finale product implementation. 


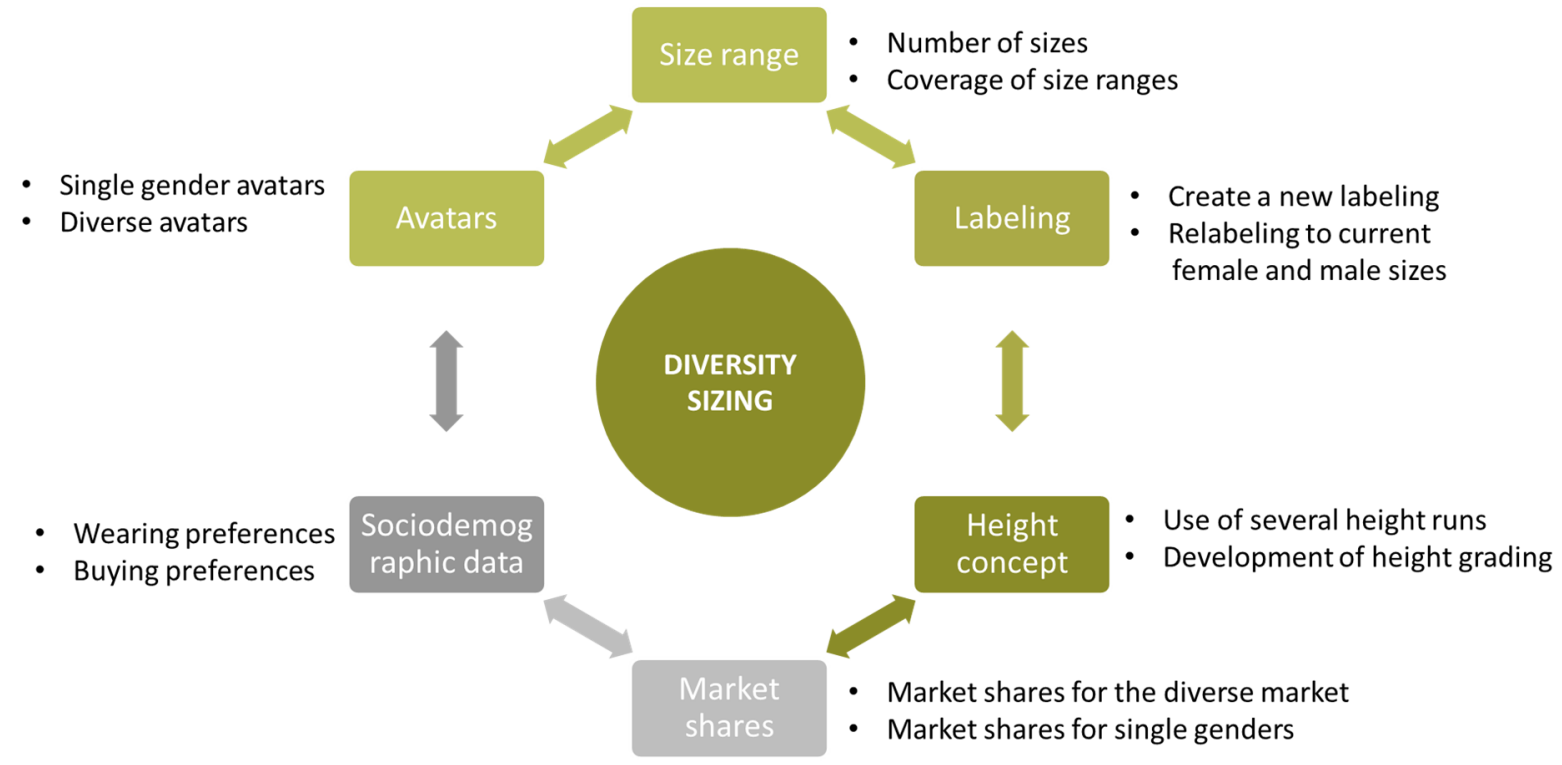

Fig. 8. Implementation of a diversity sizing: Different aspects that need to be regarded within the development of a diverse sizing approach

The diverse sizing approach should consider offering products in different sizes for covering the wide range within the market. Therefore, the optimal size range needs to be defined. Size ranges always come along with the precondition to be as wide as needed to achieve a satisfying market coverage and to be as small as possible with regards to economic efficiency. If a diversity sizing is finally developed, a feasible and gender-neutral labeling of the sizes needs to be applied. Only if the labeling is understood by both genders, the target market can respond to the developed products.

Also the final development of a length concepts needs to be evaluated as different options are possible. A diverse height grading could be applied, different height options can be offered, the conception of products with increased length tolerances or products that enable the adjustment of the product specific length dimensions based on their design are just some possible solutions.

With respect to size system development, also market share analysis is an opportunity. Market shares can show if a diverse sizing leads to a satisfying market coverage and indicate if the divers size table covers female and male data equally or if a single gender is more represented than the other. The market share calculation with regards to single sizes and single genders will show the distribution between the genders in between the diverse size system.

Another point that has been excluded from the analysis so far, is the evaluation of sociodemographic aspects, such as wearing and buying preferences of women and men. Those parameters might give important information with regards to wearing behavior and fit understanding. The results can have a significant impact on the concept of diverse collections and designs.

Also the generation of diverse avatars will play an important role within the diversity sizing approach as they represent the optimal base for product visualization on different genders with same measurements. Finding suitable fitting models is always a challenging process [12]. With regards to diverse sizing, the effort is even twice as big as at least two different models are needed for fit evaluation and testing. Based on avatars, fitting references for virtual product development can be easily generated and implemented in the process.

\subsection{Outlook on further diversity analysis}

The analysis exemplarily concentrated on the main body measurements such as the chest, waist and hip girth and the main length dimensions body height, arm length and inside leg length. Further analysis of additional secondary measurements leads to a better understanding of how differences of female and male morphology can be covered in a joint sizing.

Besides, only the European market was regarded. Depending on different markets, the results with respect to the feasibility of a diverse sizing might differ. Another aspect might be the consideration of different age groups. The analysis regarded a wide range of different age groups. A further analysis with regards to specific age groups, like for example the younger age group between 20 and 35 might lead to different results. 
This diversity analysis focused on the feasibility of combining female and male data for product development. Diversity might also include especially people in the Plus Size market as the increasing Plus Size population is often seen separately from the average population [13]. Today this part of the society is served by special curve, curvy or plus size tables, but not completely included in the regular sizing. The diversity approach can be interpreted in very different kind of ways and leads to various solutions. The general goal of a diversity sizing is to combine different people independently from their gender, nationality, physical condition or size and offer products with good fits.

All in all the analysis shows, that diversity sizing can be seen as a very interesting approach for body related product development. Many branches already combine female and male body dimensions in their product processing. For the apparel sector the implementation of female and male data is a comparably new approach. As social definitions of genders constantly change, this new understanding of gender-neutral products will be indispensable for the apparel industry. The analysis has pointed out certain challenges within the combination of male and female data, but also pointed out relevant chances and opportunities for the development of a diversity sizing that offers products with good fits to people, independently from their gender or identity.

\section{References}

[1] Messe Frankfurt Exhibition GmbH, "Diversity: „Must-have” im 21. Jahrhundert“, 2020, Available: https://texpertisenetwork.messefrankfurt.com/frankfurt/de/list/apparel-fabrics-fashion/neonytdiversity.html., accessed 2021.

[2] Vogue, S. Yotka, "The Future of Retail Is Genderless", 2020, Available: https://www.vogue.com/article/the-future-of-retail-is-genderless., accessed 2021.

[3] A. Rissiek, "Trends in body measurement and shape development - and their impact on product development, size and fit", IACDE Nordic Chapter, Stockholm, 2020.

[4] Human Solutions GmbH, "International Größen“, Workshop, Kaiserslautern, 2014.

[5] A. Rissiek and R. Trieb, "iSize - Implementation of international anthropometric survey results for worldwide sizing and fit optimization in the apparel industry", Proceeding of 3D Body scanning Conference, Lugano, Switzerland, 2010.

[6] A. Rissiek, "iSize - The international body data portal for the apparel industry", Kaiserslautern, 2021.

[7] ISO 8559:1989: "Garment construction and anthropometric surveys -- Body dimensions".

[8] ISO 7250:1996: "Basic human body measurements for technological design".

[9] Avalution $\mathrm{GmbH}$, "Is a height grading necessary and beneficial?", 2020, Kaiserslautern.

[10]EN 13402-3 (2005-04): "Size designation of clothes - Part 3: Measurements and intervals".

[11]A. Rissiek, „3D-Bodyscanning, Reihenmessungen und Avatar-generierung als Türöffner für die 3DProduktentwicklung von Bekleidung“, Kaiserslautern, 2021.

[12] A. Rissiek, "The perfect fit: brand, target group and product fit together", The New Work \& Flow, Make 3D work - end-to-end, Kaiserslautern, 2021.

[13] Human Solutions GmbH, „Große Größen für die DOB”, Workshop, Kaiserslautern, November 2015. 